\title{
Irreducible Antifield Analysis of $p$-Form Gauge Theories
}

\author{
C. Bizdadea and S. O. Saliu田 \\ Department of Physics, University of Craiova \\ 13 A. I. Cuza Str., Craiova R-1100, Romania
}

June 14, 2021

\begin{abstract}
The irreducible antifield formalism for $p$-form gauge theories with gauge invariant interaction terms is exposed. The ghosts of ghosts do not appear. The acyclicity of the Koszul-Tate operator is ensured without introducing antifields at resolution degrees higher that two.

PACS number: 11.10.Ef
\end{abstract}

It is widely known that gauge theories involving $p$-forms are important due to their link with string theory and supergravity models [1]-[6], and also due to the importance of their characteristic cohomology [7]. A typical feature of such theories is given by their redundant behavior, i.e., the reducibility of the gauge generators. The reducibility further implies the presence of ghost fields with ghost number greater that one (ghosts of ghosts, etc.), and, in the meantime, a pyramid of non-minimal variables in the framework of the Lagrangian BRST formalism [8]-[12]. The ghost and non-minimal structures of interacting $p$-forms were exposed in [13] in the light of the reducible Hamiltonian BRST method.

This paper investigates whether gauge theories involving abelian $p$-forms with interactions that possess the same gauge invariances like the free theory can be consistently quantized along the irreducible antifield-BRST formalism.

*e-mail addresses: odile.saliu@comp-craiova.ro or osaliu@central.ucv.ro 
Our main result is that this is always possible (for the priorly invoked class of theories). As far as we know, this problem has not been approached. As a consequence of our irreducible treatment, the ghosts of ghosts are not necessary, and the auxiliary fields have a linear structure (in contrast with the pyramidal reducible structure). Our method basically relies on replacing the redundant gauge theory with an irreducible one possessing the same physical observables, and on further quantizing the resulting irreducible gauge system.

The main points approached in our paper are as follows. First, we explain in detail our irreducible mechanism in the case of free abelian $p$-form gauge fields. Second, we extend the results to gauge theories involving various sorts of abelian forms with gauge invariant interaction terms, and reduce the problem to the free case analysis. This is possible by virtue of the fact that the reducibility functions associated with the interacting system are diagonal.

We start with the Lagrangian action of free abelian $p$-form gauge fields $(p>1)$

$$
S_{0_{p}}^{L}\left[A_{(p)}^{\mu_{1} \ldots \mu_{p}}\right]=-\frac{1}{2 \cdot(p+1) !} \int d^{D} x F_{(p) \mu_{1} \ldots \mu_{p+1}} F_{(p)}^{\mu_{1} \ldots \mu_{p+1}},
$$

where $F_{(p) \mu_{1} \ldots \mu_{p+1}}$ are the field strengths of the antisymmetric fields $A_{(p) \mu_{1} \ldots \mu_{p}}$ and $D>(p+1)$. The additional index $(p)$ is introduced by virtue of the subsequent discussion, where various sorts of abelian $p$-form gauge fields will be dealt with. Action (1) is invariant under the gauge transformations

$$
\delta_{\epsilon} A_{(p)}^{\mu_{1} \ldots \mu_{p}}=\partial^{\left[\mu_{1}\right.} \epsilon_{(p)}^{\left.\mu_{2} \ldots \mu_{p}\right]}=Z_{\nu_{1} \ldots \nu_{p-1}}^{\mu_{1} \ldots \mu_{p}} \epsilon_{(p)}^{\nu_{1} \ldots \nu_{p-1}}
$$

with $\left[\mu_{1} \ldots \mu_{p}\right]$ denoting antisymmetry with respect to the indices between brackets. This model is $(p-1)$-stage reducible, the reducibility relations

$$
Z_{\nu_{1} \ldots \nu_{p-k-1}}^{\mu_{1} \ldots \mu_{p-k}} Z_{\lambda_{1} \ldots \lambda_{p-k-2}}^{\nu_{1} \ldots \nu_{p-k-1}}=0
$$

holding off-shell, where the $k$ th order reducibility functions are given by

$$
Z_{\nu_{1} \ldots \nu_{p-k-1}}^{\mu_{1} \ldots \mu_{p-k}}=\frac{1}{(p-k-1) !} \partial^{\left[\mu_{1}\right.} \delta_{\nu_{1}}^{\mu_{2}} \ldots \delta_{\nu_{p-k-1}}^{\left.\mu_{p-k}\right]}, k=0, \ldots, p-1 .
$$

First, we introduce a bosonic antisymmetric field, $A_{(p)}^{\lambda_{1} \ldots \lambda_{p-k-2}}$, associated with every reducibility relation (3) corresponding to $k \geq 0$ even, and also 
a bosonic antisymmetric gauge parameter, $\epsilon_{(p)}^{\lambda_{1} \ldots \lambda_{p-k-2}}$, attached to every reducibility relation (3) corresponding to $k \geq 1$ odd. We require the next gauge transformations for the new fields

$$
\delta_{\epsilon} A_{(p)}^{\mu_{1} \ldots \mu_{p-2 k}}=\partial^{\left[\mu_{1}\right.} \epsilon_{(p)}^{\left.\mu_{2} \ldots \mu_{p-2 k}\right]}+(p-2 k+1) \partial_{\mu} \epsilon_{(p)}^{\mu \mu_{1} \ldots \mu_{p-2 k}}, k=1, \ldots, b_{p},
$$

with $b_{p}=p / 2$, or $(p-1) / 2$ for $p$ even, respectively, odd. Here and throughout this paper, we use the conventions $f^{\mu_{1} \ldots \mu_{m}}=f$ if $m=0$, and $f^{\mu_{1} \ldots \mu_{m}}=0$ if $m<0$. We consider the theory described by the Lagrangian action

$$
S_{0_{p}}^{L}\left[A_{(p)}^{\mu_{1} \ldots \mu_{p}}, A_{(p)}^{\mu_{1} \ldots \mu_{p-2 k}}\right]=S_{0_{p}}^{L}\left[A_{(p)}^{\mu_{1} \ldots \mu_{p}}\right],
$$

subject to the gauge transformations (2) and (5). It is obvious that (6) is invariant under (5).

Let us show that the above mentioned gauge transformations are irreducible. In this respect, we take $\epsilon_{(p)}^{\mu_{1} \ldots \mu_{p-2 k-1}}=\partial^{\left[\mu_{1}\right.} \theta_{(p)}^{\left.\mu_{2} \ldots \mu_{p-2 k-1}\right]}$ in (2) and (5) (for $k=0, \ldots, a_{p}$, with $a_{p}=p / 2-1$, or $(p-1) / 2$ for $p$ even, respectively, odd), and find $\delta_{\epsilon} A_{(p)}^{\mu_{1} \ldots \mu_{p}}=0$ and $\delta_{\epsilon} A_{(p)}^{\mu_{1} \ldots \mu_{p-2 k}}=(p-2 k+1) \partial_{\nu} \partial^{[\nu} \theta_{(p)}^{\left.\mu_{1} \ldots \mu_{p-2 k}\right]}$, $k>0$. The prior gauge transformations vanish if and only if $\theta_{(p)}^{\mu_{1} \ldots \mu_{p-2 k}}=$ $\partial^{\left[\mu_{1}\right.} \xi_{(p)}^{\left.\mu_{2} \ldots \mu_{p-2 k}\right]}$, so $\epsilon_{(p)}^{\mu_{1} \ldots \mu_{p-2 k-1}}=0$. In conclusion, $\delta_{\epsilon} A_{(p)}^{\mu_{1} \ldots \mu_{p-2 k}}=0$ if and only if $\epsilon_{(p)}^{\mu_{1} \ldots \mu_{p-2 k-1}}=0$. This clearly emphasizes that (8) and (5) are irreducible. In this way, we associated an irreducible theory, described by action (6) and the gauge transformations (2), (5), with the starting redundant model.

Next, we prove that both the irreducible and initial reducible theories display the same physical observables. Let $F\left(A_{(p)}^{\mu_{1} \ldots \mu_{p}}, A_{(p)}^{\mu_{1} \ldots \mu_{p-2 k}}\right)$ be an observable for the irreducible system. As any physical observable must be gauge invariant, on behalf of (2) and (1) it follows

$$
\partial_{\left[\mu_{1}\right.} \frac{\delta F}{\delta A_{(p)}^{\left.\mu_{2} \ldots \mu_{p-2 k+1}\right]}}+(p-2 k+2) \partial^{\nu} \frac{\delta F}{\delta A_{(p)}^{\nu \mu_{1} \ldots \mu_{p-2 k+1}}}=0,
$$

with $k=0, \ldots, b_{p}$. We solve the above system starting from the last equation. We explain the case $p$ even, the other situation being similar. For $k=p / 2$, equation (7) reads $\partial_{\mu} \frac{\delta F}{\delta A_{(p)}}+2 \partial^{\nu} \frac{\delta F}{\delta A_{(p)}^{\nu \mu}}=0$, which implies $\partial^{\mu} \partial_{\mu} \frac{\delta F}{\delta A_{(p)}}=0$. Because $\partial^{\mu} \partial_{\mu}$ is invertible, we obtain $\frac{\delta F}{\delta A_{(p)}}=0$, hence $\partial^{\nu} \frac{\delta F}{\delta A_{(p)}^{\nu \mu}}=0$. The next 
equation from (7), $\partial_{\left[\mu_{1}\right.} \frac{\delta F}{\delta A_{(p)}^{\left.\mu_{2} \mu_{3}\right]}}+4 \partial^{\nu} \frac{\delta F}{\delta A_{(p)}^{\nu \mu_{1} \mu_{2} \mu_{3}}}=0$, leads to $\partial^{\mu_{1}} \partial_{\left[\mu_{1}\right.} \frac{\delta F}{\delta A_{(p)}^{\left.\mu_{2} \mu_{3}\right]}}=0$, which further implies, using $\partial^{\nu} \frac{\delta F}{\delta A_{(p)}^{\nu \mu}}=0$, that $\partial^{\mu} \partial_{\mu} \frac{\delta F}{\delta A_{(p)}^{\mu_{1} \mu_{2}}}=0$, so $\frac{(p)}{\delta A_{(p)}^{\mu_{1} \mu_{2}}}=0$. Step by step, we infer along the same line that $F$ does not depend on the new fields, $\frac{\delta F}{\delta A_{(p)}^{\mu_{1} \ldots \mu_{p-2 k}}}=0$ for $k=1, \ldots, p / 2$, such that the first equation from (7) becomes $\partial_{\left[\mu_{1}\right.} \frac{\delta F}{\delta A_{(p)}^{\left.\mu_{2} \ldots \mu_{p+1}\right]}}=0$. Due to the fact that, on the one hand the last equations stand for the equation fulfilled by the observables of the reducible theory, and on the other hand $F$ depends only on the original fields, it results that any observable of the irreducible system is also an observable of the reducible one. At the same time, it is obvious that if $\bar{F}\left(A_{(p)}^{\mu_{1} \ldots \mu_{p}}\right)$ represents an observable of the reducible theory, then it remains so for the irreducible system, because it automatically satisfies (7). In consequence, the irreducible and reducible versions are physically equivalent, describing the same physical theory. From the point of view of the BRST formalism, the zeroth order cohomological groups of the longitudinal exterior derivative along the gauge orbits associated with the reducible, respectively, irreducible model coincide. Thus, if we show that the Koszul-Tate operator in the irreducible case is acyclic, the homological perturbation theory [14]-[17] ensures: (i) the nilpotency of the irreducible BRST symmetry, $s$, and (ii) $H^{0}(s)=\{$ observables , with $H^{0}(s)$ the zeroth order cohomological group of $s$. This makes legitimate from the physical point of view, i.e., from the point of view of the requirements $s^{2}=0, H^{0}(s)=\{$ observables $\}$, the replacement of the BRST quantization of the reducible theory with the quantization of the irreducible one.

At this point we briefly investigate the higher order cohomological groups of the longitudinal exterior derivative along the gauge orbits. This analysis will be relevant during the gauge-fixing process. More precisely, we show that all the higher order groups in the irreducible situation are trivial. This can be seen by introducing the minimal ghost spectrum in the irreducible case, namely $\eta_{(p)}^{\mu_{1} \ldots \mu_{p-2 k-1}}$ (with $k=0, \ldots, a_{p}$ ), and defining the action of the longitudinal exterior derivative along the gauge orbits as usually, through

$$
\begin{gathered}
D A_{(p)}^{\mu_{1} \ldots \mu_{p}}=\partial^{\left[\mu_{1}\right.} \eta_{(p)}^{\left.\mu_{2} \ldots \mu_{p}\right]} \\
D A_{(p)}^{\mu_{1} \ldots \mu_{p-2 k}}=\partial^{\left[\mu_{1}\right.} \eta_{(p)}^{\left.\mu_{2} \ldots \mu_{p-2 k}\right]}+(p-2 k+1) \partial_{\nu} \eta_{(p)}^{\nu \mu_{1} \ldots \mu_{p-2 k}}, k=1, \ldots, b_{p},
\end{gathered}
$$




$$
D \eta_{(p)}^{\mu_{1} \ldots \mu_{p-2 k-1}}=0, k=0, \ldots, a_{p} .
$$

The ghosts $\eta$ are fermionic, with pure ghost number one. From (8 9) it will follow that all the ghosts are $D$-exact. Here we indicate the line corresponding to $p$ even, the opposite situation being treated similarly. We start with the last two equations from (9), $D A_{(p)}=\partial_{\mu} \eta_{(p)}^{\mu}, D A_{(p)}^{\mu \nu}=\partial^{[\mu} \eta_{(p)}^{\nu]}+3 \partial_{\rho} \eta_{(p)}^{\rho \mu \nu}$. Applying $\partial_{\mu}$ on the last relation and using the first one, we derive $\eta_{(p)}^{\mu}=$ $D\left(\frac{1}{\square}\left(\partial_{\nu} A^{\nu \mu}+\partial^{\mu} A\right)\right)$, such that $\partial_{\rho} \eta_{(p)}^{\rho \mu \nu}=\frac{1}{3} D\left(\frac{1}{\square} \partial_{\rho} \partial^{[\rho} A_{(p)}^{\mu \nu]}\right)$. Multiplying the next equation from (9) by $\partial_{\mu}$ and taking into account the expression of $\partial_{\rho} \eta_{(p)}^{\rho \mu \nu}$, we obtain $\eta_{(p)}^{\mu \lambda \rho}=D\left(\frac{1}{\square}\left(\partial_{\nu} A^{\nu \mu \lambda \rho}+\frac{1}{3} \partial^{[\mu} A^{\lambda \rho]}\right)\right)$. Acting like before, we infer

$$
\eta_{(p)}^{\mu_{1} \ldots \mu_{p-2 k-1}}=D\left(\frac{1}{\square} \chi_{(p)}^{\mu_{1} \ldots \mu_{p-2 k-1}}\right), k=0, \ldots, a_{p},
$$

with $\chi_{(p)}^{\mu_{1} \ldots \mu_{p-2 k-1}}=\partial_{\mu} A_{(p)}^{\mu \mu_{1} \ldots \mu_{p-2 k-1}}+\frac{1}{p-2 k-1} \partial^{\left[\mu_{1}\right.} A_{(p)}^{\left.\mu_{2} \ldots \mu_{p-2 k-1}\right]}$. On the one hand, from (10) it follows that any $D$-closed quantity of pure ghost number greater than zero is a polynomial in the ghosts with coefficients that are gauge invariant functions. On the other hand, from (11) it results that any such polynomial is $D$-exact. As a consequence, all $H^{q}(D)$ with $q>0$ vanish in our irreducible approach.

Now, we prove that the Koszul-Tate operator corresponding to the irreducible model, $\delta$, is truly acyclic. Accordingly the standard BRST receipt, the minimal antifield spectrum reads as $A_{(p) \mu_{1} \ldots \mu_{p-2 k}}^{*}\left(k=0, \ldots, b_{p}\right)$ and $\eta_{(p) \mu_{1} \ldots \mu_{p-2 k-1}}^{*}\left(\right.$ with $\left.k=0, \ldots, a_{p}\right)$. The $A^{*}$ s are fermionic and have antighost number one, while the $\eta^{*}$ 's are bosonic, with antighost number two. We define the action of $\delta$ on the generators from the BRST complex as usually by

$$
\begin{gathered}
\delta A_{(p)}^{\mu_{1} \ldots \mu_{p-2 k}}=0, k=0, \ldots, b_{p}, \delta \eta_{(p)}^{\mu_{1} \ldots \mu_{p-2 k-1}}=0, k=0, \ldots, a_{p}, \\
\delta A_{(p) \mu_{1} \ldots \mu_{p}}^{*}=-\frac{1}{p !} \partial^{\nu} F_{(p) \nu \mu_{1} \ldots \mu_{p}}, \\
\delta A_{(p) \mu_{1} \ldots \mu_{p-2 k}}^{*}=0, k=1, \ldots, b_{p}, \\
\delta \eta_{(p) \mu_{1} \ldots \mu_{p-2 k-1}}^{*}=-\left((p-2 k) \partial^{\mu} A_{(p) \mu \mu_{1} \ldots \mu_{p-2 k-1}}^{*}+\partial_{\left[\mu_{1}\right.} A_{\left.(p) \mu_{2} \ldots \mu_{p-2 k-1}\right]}^{*}\right) .
\end{gathered}
$$

From (13) and (14), we observe that $\partial^{\mu_{1}} A_{(p) \mu_{1} \ldots \mu_{p}}^{*}$ and $A_{(p) \mu_{1} \ldots \mu_{p-2 k}}^{*}$ are $\delta$-closed quantities. This implies that there can exist $\delta$-closed polynomials in the above 
objects with coefficients that may involve the $A_{(p)}^{\mu_{1} \ldots \mu_{p-2 k}}$ 's in the higher order resolution degree homological groups of $\delta$. In the reducible BRST approach, the $\delta$-closed polynomials are killed step by step in the homology of $\delta$ by appropriately introducing some new generators (the antifields associated with the ghosts of ghosts). This is not necessary in our treatment, as we show that the $\delta$-closed quantities with positive resolution degrees from the irreducible case are $\delta$-exact. In this respect, we prove that $\partial^{\mu_{1}} A_{(p) \mu_{1} \ldots \mu_{p}}^{*}$ and $A_{(p) \mu_{1} \ldots \mu_{p-2 k}}^{*}$ are $\delta$-exact. The proof will be exemplified in the case $p$ even, being similar if $p$ odd, and goes as follows. We start with the last equation from (15), $\delta \eta_{(p) \mu_{1}}^{*}=$ $-\left(2 \partial^{\mu} A_{(p) \mu \mu_{1}}^{*}+\partial_{\mu_{1}} A_{(p)}^{*}\right)$, that leads to $\delta\left(\partial^{\mu} \eta_{(p) \mu}^{*}\right)=-\partial^{\mu} \partial_{\mu} A_{(p)}^{*}$, so $A_{(p)}^{*}=$ $\delta\left(\frac{-1}{\square} \partial^{\mu} \eta_{(p) \mu}^{*}\right)$. Thus, we have that $\partial^{\mu} A_{(p) \mu \mu_{1}}^{*}=\frac{1}{2} \delta\left(\frac{1}{\square} \partial_{\mu_{1}} \partial^{\mu} \eta_{(p) \mu}^{*}-\eta_{(p) \mu_{1}}^{*}\right)$. Using the last relation in the next equation from (15) multiplied by $\partial^{\mu_{1}}$, we find $A_{(p) \mu_{1} \mu_{2}}^{*}=\delta\left(\frac{-1}{\square}\left(\frac{1}{2} \partial_{\left[\mu_{1}\right.} \eta_{\left.(p) \mu_{2}\right]}^{*}+\partial^{\mu} \eta_{(p) \mu_{1} \mu_{2}}^{*}\right)\right)$. Along the same line, we derive

$$
A_{(p) \mu_{1} \ldots \mu_{p-2 k}}^{*}=\delta\left(\frac{-1}{\square}\left(\frac{1}{p-2 k} \partial_{\left[\mu_{1}\right.} \eta_{\left.(p) \mu_{2} \ldots \mu_{p-2 k}\right]}^{*}+\partial^{\mu} \eta_{(p) \mu \mu_{1} \ldots \mu_{p-2 k}}^{*}\right)\right),
$$

for $k=1, \ldots, \frac{p}{2}$, and

$$
\partial^{\mu} A_{(p) \mu \mu_{1} \ldots \mu_{p-1}}^{*}=\delta\left(\frac{-1}{p \square}\left(\partial^{\mu} \partial_{[\mu} \eta_{\left.(p) \mu_{1} \ldots \mu_{p-1}\right]}^{*}\right)\right) .
$$

Formulas (16 17) restore the $\delta$-exactness of the investigated quantities. Moreover, we cannot find $\delta$-closed expressions involving $\eta_{(p) \mu_{1} \ldots \mu_{p-2 k-1}}^{*}$ due to the irreducibility of the gauge transformations (2) and (5). In conclusion, the above antifield spectrum is enough to enforce the acyclicity of $\delta$, so we do not need to introduce antifields with resolution degrees higher that two. In this way, the acyclicity of $\delta$ is fully guaranteed within our irreducible approach, hence, as explained above, we can replace the quantization of the reducible model with the one of the irreducible theory.

In the sequel we perform the antifield-BRST quantization of the irreducible system built previously. With the minimal ghost and antifield spectra at hand, we choose the non-minimal sector $\left(\bar{\eta}_{(p)}^{\mu_{1} \ldots \mu_{p-2 k-1}}, \bar{\eta}_{(p) \mu_{1} \ldots \mu_{p-2 k-1}}^{*}\right)$, $\left(B_{(p)}^{\mu_{1} \ldots \mu_{p-2 k-1}}, B_{(p) \mu_{1} \ldots \mu_{p-2 k-1}}^{*}\right)$ with $k=0, \ldots, a_{p}$. The $B$ 's and $\bar{\eta}^{*}$ 's are bosonic and with ghost number zero, while the remaining variables are fermionic, of 
ghost number minus one. The ghost number is defined as the difference between the pure ghost number and the antighost number. The non-minimal solution of the master equation is expressed by

$$
\begin{aligned}
& S^{(p)}=S_{0_{p}}^{L}+\int d^{D} x\left(A_{(p) \mu_{1} \ldots \mu_{p}}^{*} \partial^{\left[\mu_{1}\right.} \eta_{(p)}^{\left.\mu_{2} \ldots \mu_{p}\right]}+\sum_{k=0}^{a_{p}} \bar{\eta}_{(p) \mu_{1} \ldots \mu_{p-2 k-1}}^{*} B_{(p)}^{\mu_{1} \ldots \mu_{p-2 k-1}}\right. \\
& \left.+\sum_{k=1}^{b_{p}} A_{(p) \mu_{1} \ldots \mu_{p-2 k}}^{*}\left(\partial^{\left[\mu_{1}\right.} \eta_{(p)}^{\left.\mu_{2} \ldots \mu_{p-2 k}\right]}+(p-2 k+1) \partial_{\mu} \eta_{(p)}^{\mu \mu_{1} \ldots \mu_{p-2 k}}\right)\right)
\end{aligned}
$$

In order to fix the gauge, we recall formula (11), which actually shows how the ghosts remove the unphysical degrees of freedom. Indeed, we can regard the functions $\chi_{(p)}^{\mu_{1} \ldots \mu_{p-2 k-1}}$ like some fields with the gauge transformations $\delta_{\bar{\epsilon}} \chi_{(p)}^{\mu_{1} \ldots \mu_{p-2 k-1}}=\bar{\epsilon}_{(p)}^{\mu_{1} \ldots \mu_{p-2 k-1}}$, where $\bar{\epsilon}_{(p)}^{\mu_{1} \ldots \mu_{p-2 k-1}}=\square \epsilon_{(p)}^{\mu_{1} \ldots \mu_{p-2 k-1}}$, so the $\chi_{(p)}$ 's are purely gauge. Then, it is natural to take the gauge conditions $\chi_{(p)}^{\mu_{1} \ldots \mu_{p-2 k-1}}=0$ in the gauge-fixing process. They are enforced via the gaugefixing fermion

$$
\psi_{(p)}=\sum_{k=0}^{a_{p}} \int d^{D} x \bar{\eta}_{(p) \mu_{1} \ldots \mu_{p-2 k-1}} \chi_{(p)}^{\mu_{1} \ldots \mu_{p-2 k-1}},
$$

from which we derive the gauge-fixed action

$$
\begin{aligned}
& S_{\psi_{(p)}}^{(p)}=S_{0_{p}}^{L}+\sum_{k=0}^{a_{p}} \int d^{D} x\left(\bar{\eta}_{(p) \mu_{1} \ldots \mu_{p-2 k-1}} \square \eta_{(p)}^{\mu_{1} \ldots \mu_{p-2 k-1}}+\right. \\
& \left.B_{(p) \mu_{1} \ldots \mu_{p-2 k-1}} \chi_{(p)}^{\mu_{1} \ldots \mu_{p-2 k-1}}\right) .
\end{aligned}
$$

It is easy to check that the gauge-fixed action (20) possesses no residual gauge invariances. The formula (20) is the final output of our irreducible procedure for abelian free $p$-form gauge fields. It establishes that one can consistently quantize free abelian $p$-forms without introducing either ghosts of ghosts or their antifields. At the same time, we remark that our approach outlines some good gauge conditions with a direct physical content. These conditions appear in the reducible procedure from the necessity of implementing some irreducible Lorentz type gauge conditions.

As can be seen from (5), our procedure activates some of the ineffective gauge transformations implied within the reducible treatment. Then, it appears legitimate the question whether we can make effective all the ineffective 
gauge transformations. The answer is however negative. We show this in the simple case of three-form gauge fields, the general proof following a similar line. The gauge invariances of abelian three-forms, $\delta_{\epsilon} A_{(3)}^{\mu \nu \rho}=\partial^{[\mu} \epsilon_{(3)}^{\nu \rho]}$, become ineffective if one takes $\epsilon_{(3)}^{\nu \rho}=\partial^{[\nu} \epsilon_{(3)}^{\rho]}$ and further $\epsilon_{(3)}^{\rho}=\partial^{\rho} \epsilon_{(3)}$. Accordingly our method, only the gauge transformations with the parameters $\epsilon_{(3)}^{\rho}=\partial^{\rho} \epsilon_{(3)}$ become effective via $\delta_{\epsilon} A_{(3)}^{\mu}=\partial^{\mu} \epsilon_{(3)}+2 \partial_{\nu} \epsilon_{(3)}^{\nu \mu}$. In order to make also effective the transformations with the gauge parameters $\epsilon_{(3)}^{\nu \rho}=\partial^{[\nu} \epsilon_{(3)}^{\rho]}$, it is necessary to add the new fields $\left(A_{(3)}^{\mu \nu}, A_{(3)}\right)$ with the gauge variations $\delta_{\epsilon} A_{(3)}^{\mu \nu}=\partial^{[\mu} \epsilon_{(3)}^{\nu]}$, respectively, $\delta_{\epsilon} A_{(3)}=\partial_{\nu} \epsilon_{(3)}^{\nu}$. The gauge transformations of the theory with three-, two-, one-, and zero-form gauge fields are irreducible. However, this new irreducible system does not describe the same physical observables like those of free abelian three-form gauge fields. This can be seen by writing down the equations associated with the physical observables of the irreducible theory, namely, $3 \partial^{\mu} \frac{\delta F}{\delta A_{(3)}^{\mu \nu \rho}}+\partial_{[\nu} \frac{\delta F}{\delta A_{(3)}^{\rho}}=0,2 \partial^{\mu} \frac{\delta F}{\delta A_{(3)}^{\mu \nu}}+\partial_{\nu} \frac{\delta F}{\delta A_{(3)}}=0, \partial^{\mu} \frac{\delta F}{\delta A_{(3)}^{\mu}}=0$. From the first and third equations, we infer $\partial^{\mu} \frac{\delta F}{\delta A_{(3)}^{\mu \nu \rho}}=0, \frac{\delta F}{\delta A_{(3)}^{\mu}}=0$, while the second relation implies $\frac{\delta F}{\delta A_{(3)}}=0$, and $\partial^{\mu} \frac{\delta F}{\delta A_{(3)}^{\mu \nu}}=0$. The last equation does not lead to $\frac{\delta F}{\delta A_{(3)}^{\mu \nu}}=0$, so the observables of the irreducible theory do not coincide with those of the reducible one. We can remove this deficiency by adding a new gauge parameter $\epsilon_{(3)}^{\mu \nu \rho}$, and taking the gauge transformations of $A_{(3)}^{\mu \nu}$ under the form $\delta_{\epsilon} A_{(3)}^{\mu \nu}=\partial^{[\mu} \epsilon_{(3)}^{\nu]}+3 \partial_{\rho} \epsilon_{(3)}^{\rho \mu \nu}$. In this situation, the above equations fulfilled by the observables must be supplemented with $\partial_{[\rho} \frac{\delta F}{\delta A_{(3)}^{\mu \nu}}=0$. Applying $\partial^{\rho}$ on the last relation, and using $\partial^{\mu} \frac{\delta F}{\delta A_{(3)}^{\mu \nu}}=0$, it follows $\frac{\delta F}{\delta A_{(3)}^{\mu \nu}}=0$, as required. Hence, the supplementary gauge parameters $\epsilon_{(3)}^{\mu \nu \rho}$ helps us at recovering the equivalence between the original and the new model at the level of physical observables. A new problem appears now, namely, the acyclicity of the Koszul-Tate operator. Indeed, $\delta$ is no longer acyclic. On behalf of the definitions of $\delta$ acting on the antifields, we find at the resolution degree equal with two the $\delta$-closed quantities $\partial^{\lambda} \partial_{[\lambda} \eta_{(3) \mu \nu \rho]}^{*}$, where $\eta_{(3) \mu \nu \rho}^{*}$ denote the antifields corresponding to the gauge parameters $\epsilon_{(3)}^{\mu \nu \rho}$. In change, these quantities are not $\delta$-exact. This signalizes that the part of the gauge transformations for $A_{(3)}^{\mu \nu}$ involving the parameters $\epsilon_{(3)}^{\mu \nu \rho}$ is vanishing under the change $\epsilon_{(3)}^{\mu \nu \rho}=\partial_{\lambda} \partial^{[\lambda} \theta_{(3)}^{\mu \nu \rho]}$ with arbitrary non-vanishing $\theta_{(3)}^{\mu \nu \rho}$,s, which means that some reducibility is present. Thus, if we try to make physically equiv- 
alent the two theories, then we lose the irreducibility, and, conversely, if we ensure the irreducibility, the two systems are no longer physically equivalent. In consequence, one cannot make effective all the ineffective gauge transformations, and, at the same time, enforce the irreducibility. This argues the introduction only of the fields $A_{(p)}^{\mu_{1} \ldots \mu_{p-2 k}}$ with the gauge transformations (5).

With the above analysis at hand, we are ready to investigate the irreducible BRST quantization of gauge theories with abelian $p$-form gauge fields involving interacting terms that are gauge invariant under (2). These terms may increase the derivative order of the field equations because the Lagrangian density contains only the field strengths and their derivatives. 1 For definiteness, we begin with a gauge theory described by the action

$$
S_{0}^{L}\left[\left(A_{\left(p_{a}\right)}^{\mu_{1} \ldots \mu_{p_{a}}}\right)\right]=\sum_{a} S_{0_{p_{a}}}^{L}\left[A_{\left(p_{a}\right)}^{\mu_{1} \ldots \mu_{p_{a}}}\right]+S_{I}^{L}\left[\left(A_{\left(p_{a}\right)}^{\mu_{1} \ldots \mu_{p_{a}}}\right)\right],
$$

where $a=1, \ldots, n$ and $p_{a} \geq 1$. Here, $A_{\left(p_{a}\right)}^{\mu_{1} \ldots \mu_{p_{a}}}$ represents an abelian $p_{a}$-form, $S_{0_{p_{a}}}^{L}\left[A_{\left(p_{a}\right)}^{\mu_{1} \ldots \mu_{p_{a}}}\right]$ is of the type (1), and $S_{I}^{L}$ involves all the consistent interaction terms invariant under the gauge transformations of the type (2) for every $p_{a}$. The theory described by (21) can be quantized in an irreducible manner accordingly the approach to free $p$-forms discussed earlier. In this light, for every $p_{a}>1$ (abelian one-forms are irreducible) we add the fields $A_{\left(p_{a}\right)}^{\mu_{1} \ldots \mu_{p_{a}-2 k}}, k=1, \ldots, b_{p_{a}}$, the gauge parameters $\epsilon_{\left(p_{a}\right)}^{\mu_{1} \ldots \mu_{p_{a}-2 k-1}}, k=1, \ldots, a_{p_{a}}$, and require the gauge transformations of the type (5). Although we investigate an interacting theory, the analysis goes almost identically with the free case for every sort of original fields because the reducibility functions of the interacting theory contain diagonal blocks. The only difference resides in the action of the Koszul-Tate operator on the initial fields, as the interaction terms may add some new terms to the free equations of motion. This does not affect the analysis from the free case, as the new equations of motion satisfy the same Noether identities like in the absence of interaction. Hence, the proof of the physical equivalence (at the level of observables) between the reducible and irreducible theories, respectively, the acyclicity of the irreducible Koszul-Tate operator remains unchanged. With these considerations at hand, it is simply to see that the gauge-fixed action reads

$$
S_{\psi}=S_{0}^{L}\left[\left(A_{\left(p_{a}\right)}^{\mu_{1} \ldots \mu_{p_{a}}}\right)\right]+\sum_{a} S_{\psi_{p_{a}}}^{\left(p_{a}\right)} .
$$

\footnotetext{
${ }^{1}$ In certain dimensions, one can add topologically interactions. Such interactions are not strictly gauge invariant, but only invariant up to some surface terms.
} 
In (22) $S_{\psi_{p_{a}}}^{\left(p_{a}\right)}$ is expressed by the second and third terms in the right-hand side of (20), with $p \rightarrow p_{a}$. The gauge-fixing fermion on account of which we reach (22) is given by $\psi=\sum_{a} \psi_{p_{a}}$, with $\psi_{p_{a}}$ like in (19). The derivation of the gaugefixed action (22) completes our treatment. At this point, we mention that our irreducible Lagrangian investigation of interacting $p$-form gauge theories is advantageous as compared with a Hamiltonian analysis because $S_{I}^{L}$ may contain higher-order derivative terms which overwhelm both the canonical approach and the construction of some irreducible first-class constraints.

To conclude with, in this paper we proved that gauge theories with abelian $p$-form gauge fields can be quantized along an irreducible antifield BRST fashion. The cornerstone of our approach is given by the construction of an irreducible gauge theory in a way that makes legitimate the replacement of the reducible antifield-BRST quantization with the irreducible one. The acyclicity of the irreducible Koszul-Tate operator was explicitly emphasized, and also the equivalence between the irreducible and reducible theories at the level of physical observables was completely elucidated. At the same time, our formalism leads to some gauge conditions allowing a meaningful physical interpretation. Our results will be used in a next paper at the investigation of the deformation of the master equation [18 for gauge theories with abelian $p$-forms, and also at solving appropriately some cohomological aspects linked with such theories.

\section{References}

[1] V. I. Ogievetski, I. V. Polubarinov, Sov. J. Nucl. Phys. 4 (1967) 156

[2] E. Cremmer, J. Scherk, Nucl. Phys. B72 (1974) 117

[3] M. Kalb, P. Ramond, Phys. Rev. D9 (1974) 2273

[4] C. Teitelboim, Phys. Lett. 167B (1986) 63; 69

[5] A. Lahiri, Mod. Phys. Lett. A8 (1993) 2403 and references therein

[6] M. B. Green, J. H. Schwarz, E. Witten, Superstring Theory (Cambridge University Press, Cambridge, 1987) 
[7] M. Henneaux, B. Knaepen, C. Schomblond, Commun. Math. Phys. 186 (1997) 137

[8] I. A. Batalin, G. A. Vilkovisky, Phys. Lett. B102 (1981) 27

[9] I. A. Batalin, G. A. Vilkovisky, Phys. Rev. D28 (1983) 2567

[10] I. A. Batalin, G. A. Vilkovisky, J. Math. Phys. 26 (1985) 172

[11] M. Henneaux, Nucl. Phys. B (Proc. Suppl.) 18A (1990) 47

[12] M. Henneaux, C. Teitelboim, Quantization of Gauge Systems, Princeton Univ. Press, Princeton, 1992

[13] L. Baulieu, M. Henneaux, Nucl. Phys. B277 (1986) 268

[14] G. Hirsch, Bull. Soc. Math. Belg. 6 (1953) 79

[15] J. D. Stasheff, Trans. Amer. Math. Soc. 108 (1963) 215; 293

[16] V. K. A. M. Gugenheim, J. Pure Appl. Alg. 25 (1982) 197

[17] V. K. A. M. Gugenheim, J. D. Stasheff, Bull. Soc. Math. Belg. 38 (1986) 237

[18] G. Barnich, M. Henneaux, Phys. Lett. B311 (1993) 123 